\title{
Homology Modeling, Molecular Dynamics Simulation and Essential Dynamics on Anopheles gambiae D7r1
}

\author{
Chayanika Goswami, Manikandan Jayraman, Tamizhmathi Bakthavachalam, \\ Guneswar Sethi, Ramadas Krishna*
}

Centre for Bioinformatics, School of Life Sciences, Pondicherry University, India

Copyright $\bigcirc 2017$ by authors, all rights reserved. Authors agree that this article remains permanently open access under the terms of the Creative Commons Attribution License 4.0 International License

\begin{abstract}
The proteins produced by the saliva and salivary glands of blood sucking arthropods play a vital role in transmission of the infected parasite to host and interfere the parasitic life cycle. The structure prediction of D7r1 and its active site leads to inhibition of hemostasis and inflammation in the host. D7r1 is a member of D7-related (D7r) salivary gland proteins that interferes various aspects of host physiology. Considering the significance of a protein, three dimensional structure of D7r1 model was generated by homology modeling and validated by PROCHECK, ERRAT, Verify-3D, RAMPAGE and Q mean server. The predicted structure has $96.9 \%$ of residues in the most favored region of the Ramachandran plot. The sequence and structural alignment between $\mathrm{D} 7 \mathrm{r} 1$ and template $2 \mathrm{PQL}$ reveals that similar active site residues such as Ile42, Arg43, Tyr45, His56, Met57, Val60, Phe131, and Met156 involved in binding pocket formation. Further, a molecular dynamics simulation study was performed to reveal the prolonged stability of $\mathrm{D} 7 \mathrm{r} 1$ protein. The essential dynamics which include PCA and FEL analysis were used to evaluate the conformational stability of D7r1. This combined molecular dynamics simulation and essential dynamics were used to provide comprehensive information of $\mathrm{D} 7 \mathrm{r} 1$ and its active site prediction gain insights into the development of novel lead molecules for disrupting host-seeking behavior of mosquitoes.
\end{abstract}

Keywords Anopheles gambiae, D7-related (D7r) Proteins, Homology Modeling, Molecular Dynamics Simulation, PCA and FEL Analysis

\section{Introduction}

The blood feeding insects are responsible for transmitting a number of potentially lethal diseases like encephalitis, yellow fever and malaria (a most deadly tropical disease) are considered to be an important reason for socio-economic burden in developing countries [1]. In 2016, World Health Organization (WHO) reported the death of 438,000 people out of 214 million people with malarial infection. The deceased people were mainly children under the age of 5 [2]. The African mosquito Anopheles gambiae, carries the pathogen Plasmodium falciparum in their saliva and responsible for transmitting malaria to humans. The multi-drug resistance capability of Plasmodium parasites as well as resistance against chemotherapeutic agents makes it imperative to develop new anti-malarial drug agents [3]. Researchers are currently focused on developing new drugs and vaccines against the parasite. Till date, the most successful tactic to control malarial transmission to humans is found to be a reduction of the frequency of contact between the mosquito vectors and their human targets [4].

The African mosquito Anopheles gambiae is considered the best studied malarial parasite of Plasmodium falciparum and also plays a crucial role in the parasite life cycle. Approximately, 450 species of Anopheles mosquitoes are available worldwide, among them 60 species are reported to be a vector of human malaria [5]. Parasites are transmitted to humans once Anopheles mosquito bites and subsequently destroy red blood cells by multiplying in the host. Predominantly, female Anopheles gambiae mosquitos have an extremely high preference for feeding on humans. The selectivity of the female mosquito for human host is guided by olfactory responses to odor molecules that emanate from human skin and sweat [6]. The salivary gland of blood-feeding arthropods contains various potent bioactive substances essential for blood feeding. Several proteins produced by the salivary glands are significant for overcoming the problems caused by the host such as itch responses, immune defenses and homeostasis [7]. Further, it has been revealed that the pharmacological activity of arthropod salivary interrupts malarial pathogen transmission. Proteomic studies were performed on mosquito salivary gland proteins to define their imperative role on Plasmodium falciparum [8].

The identification of 67 proteins from Anopheles gambiae 
salivary glands by Kalume et al.. [9] has been an initial step towards the grouping of numerous proteins and peptides in the salivary proteome. Earlier studies reports that the saliva from female Anopheles gambiae has several antihemostatic proteins that include the platelet aggregation inhibitor apyrase, anophelin, and catechol oxidase-peroxidase. Of these, the D7-related (D7r) proteins are known to interfere with various aspects of host physiology and are divided into a group of five polypeptides (D7r1-D7r5) [10]. Further, recent study confirms that five major salivary gland proteins such as a putative 5 '-nucleotidase, anti-platelet protein, long form D7 salivary protein, D7r1 and gSG6 from $A n$. campestris mosquitoes involved in blood feeding [11]. Since, saliva proteins are antigenic and immunogenic which enhances the infectivity of the parasite and its immunomodulatory, anti-inflammatory and anti-coagulant properties helps in successful blood feeding [12].

D7r1 protein also known as hamadarin, exhibits anticoagulant or antikinin activity by forming strong interactions with high molecular weight kininogen and factor XIIa, both are considered to be important components of the contact activation system of coagulation [3, 13]. Moreover, It has been proven that $\mathrm{D} 7 \mathrm{r}$ (D7r1-D7r4) proteins bind to biogenic amines such as serotonin, norepinephrine and histamine, accordingly reduces the concentrations of these effectors at blood feeding site [3]. Surprisingly, D7r5 protein is not involved in any strong binding interaction with biogenic amines. The sequence comparison among $\mathrm{D} 7 \mathrm{r}$ protein families (D7r1-D7r5) illustrates a possible relationship among the $\mathrm{D} 7 \mathrm{r}$ proteins and may function as scavengers of biogenic amines [14].

Owing to the importance of D7r1 as a potent antimalarial drug target, the comparative based homology modeling was performed to generate 3D models of $\mathrm{D} 7 \mathrm{r} 1$ by using MODELLER 9v7 suite. Several standalone tools and servers were used to evaluate the reliability of predicted model and indicating the high quality of the model. Further, the modeled structure was minimized and equilibrated by Molecular Dynamics simulation (MDS) for period of $50 \mathrm{~ns}$ to ensure the structure stability. Additionally, the Principal Component Analysis (PCA) and Free Energy Landscape (FEL) were used to investigate the functional dynamics and conformational changes in $\mathrm{D} 7 \mathrm{r} 1$ protein.

\section{Materials and Methods}

\subsection{Protein Sequence Information}

The primary protein sequence of $\mathrm{D} 7 \mathrm{r} 1$ protein is retrieved from the Uniprot database (UniProtKB accession number: Q9UB30) in FASTA format. The ProtParam tool in the ExPASy server [15] was used to calculate the physio-chemical properties of $\mathrm{D} 7 \mathrm{r} 1$. The secondary structure of D7r1 is predicted by PSIPRED [16] and analyzed by SOPMA server [17].

\subsection{Disordered Region Prediction}

The high flexibility and disordered regions of D7r1 protein were determined by GlobPlot [18].

\subsection{Homology Modeling of D7r1}

Comparative modeling or Homology modeling has become an efficient approach for predicting the three dimensional structure of an unknown protein based on template sequence alignment. The homology modeling of D7r1 was carried out by MODELLER (9v11) suite [19] and the alignment of target and template proteins were performed by Clustal Omega [20]. PyMOL [21] and Chimera [22] were used for visualization.

\subsection{Structure Validation of D7r1}

The stereo chemical validation and the reliability of predicted model were carried out using various online tools. RAMPAGE [23] server, assessed the stereo chemical quality of protein by building Ramachandran Plot of the protein backbone structure. The structure was further subjected to Structural Analysis and Verification Server (SAVES) [24] which includes PROCHECK [25] and ERRAT [26] to evaluate the reliability of protein. In addition, the structure was given to Q MEAN (Qualitative Model Energy ANalysis) [27] server to calculate the overall quality of the protein by comparing experimentally solved reference structures. The Z-score calculated by ProSA [28] web server is used to inspect the overall quality of the protein. Further, the quantitative evaluation of computationally modeled D7r1 structure was carried out in PROSESS (PROtein Structure Evaluation Suite and Server) [29] which uses VADAR (Volume Area Dihedral Angle Reporter) [30] web tool to identify global and residue specific problem.

\subsection{Molecular Dynamics Simulation}

The structural behavior and conformational motion of modeled D7r1 was studied by molecular dynamics simulation using GROMACS package version 4.5.3 [31, 32]. The protein topology was built by GROMOS96 43a1 force field [33] and the protein model was solvated with SPC216 water model in a cubic box size of $1.50 \mathrm{~nm}$. The system was surrounded with simple point charge (SPC) and neutralized by adding 10 chlorine $\left(\mathrm{Cl}^{-}\right)$ions. The periodic boundary conditions were applied in all directions to avoid edge effects. Prior to MD simulation, the system was subjected to two rounds of energy minimization with maximum steps of 50,000 for the modeled protein using steepest descent algorithm, followed by conjugate gradient algorithm with a tolerance of $1000 \mathrm{kJmol}^{-1} \mathrm{~nm}^{-1}$. The energy minimized systems was equilibrated at both NVT (constant volume and normal temperature) and NPT (constant pressure and normal temperature) ensembles for $150 \mathrm{ps}$. The Particle Mesh Ewald 
(PME) [34] summation was used for long range electrostatic interactions with a PME order of 4 and a Fourier spacing of $0.16 \mathrm{~nm}$. The LINCS algorithm [32] was used for constraining the bonds containing hydrogen atoms and SETTLE algorithm [35] was used to constrain the geometry of water molecules. V-rescale weak coupling method was used to regulate the temperature $(300 \mathrm{~K})$ and ParrinelloRahman method [36] was used to set the pressure (at 1atm). Finally, the MD run of $50 \mathrm{~ns}$ time scale was carried out with a time step of 2 femtosecond (fs) and the simulated model was also validated for their structure quality using above mentioned servers.

\subsection{Principal Components and Free Energy Landscape Analysis}

PCA is an effective method for finding global and correlated motions by obtaining standard information from the MD trajectories through dimensionality reduction method $[37,38]$. PCA was used to distinguish the large scale collective motions from the random thermal fluctuations. These large scale collective motions are responsible for determining the functional state of a protein [39].

PCA method is based on the calculation and diagonalization of the covariance matrix. The elements $C_{i j}$ in this matrix is defined by [40].

$$
\mathrm{C}_{\mathrm{ij}}=\left(\mathrm{x}_{\mathrm{i}}-\mathrm{X}_{\mathrm{i}}\right)\left(\mathrm{x}_{\mathrm{j}}-\mathrm{x}_{\mathrm{j}}\right)
$$

Where, $\mathrm{x}_{\mathrm{i}}\left(\mathrm{x}_{\mathrm{j}}\right)$ is the coordinate of the ith (jth) atom and represents an ensemble average. In general, the few initial principal components (PCs) are responsible for the most important conformational changes of bimolecular system by storing eigenvectors with the highest eigenvalues. The g_covar module of GROMACS package $[31,32]$ was used to generate and diagnolize the covariance matrix. Meanwhile, g_anaeig module was used to calculate the eigenvalues and eigenvectors. The eigenvectors of the matrix represent the directions of the motions and the eigenvalues describes the magnitude of the motion for each direction. The cosine content $\left(\mathrm{C}_{\mathrm{i}}\right)$ value of PCs is considered to be an important measure to differentiate the PCs that captured the non-random fluctuations from the collective motions. Normally, the cosine content value varies between 0 (no cosine) and 1 (perfect cosine) in the total simulation time period (T):

$$
C_{i}=\frac{2}{T}\left(\int \cos (i \pi t) p_{i}(t) d t\right)^{2}\left(\int p_{i}^{2}(t) d t\right)^{-1}
$$

In general, the protein dynamics can be defined based on their initial eigenvector cosine contribution. However, in most of the cases the cosine content value of first eigenvector is near to 1 , which normally represents large scale motions in the protein simulation system. Therefore, these large scale motions cannot be used as a measure for describing the protein structural behaviour in terms of the free energy landscape (FEL). The FEL can be constructed on the basis of PCA [40] and provide a quantitative description of protein dynamics. In this study, PCA was performed in order to investigate the functional motions of D7r1 by extracting the top 20 PCs (Projection eigenvectors). In which, the cosine content less than 0.2 were used as suitable reaction coordinates (PC1 and PC2) to generate the FEL energy graph by the g_sham module of GROMACS. The FEL analysis gives descriptive information on dominant conformations with lowest energy and provides their structural transition during the simulation period.

\subsection{Active Site Prediction}

Active site, also called catalytic site of protein consisting of amino acid residues act as a donor or acceptor for ligand or lead molecules. Identification and characterization of the protein active site is a crucial step in molecular docking studies. The active site of $\mathrm{D} 7 \mathrm{r} 1$ protein was predicted by both computational approach and literature studies. The $\mathrm{COACH}$ server which contains COFACTOR [41], ConCavity [42] and S-SITE [43] are used to predict the possible ligand binding site or binding pocket of $\mathrm{D} 7 \mathrm{r} 1$ protein.

\section{Results and Discussion}

\subsection{Primary and Secondary Structure Analysis}

\begin{tabular}{|c|c|c|}
\hline Amino acid name & Amino acid No & $\begin{array}{c}\text { Amino acid } \\
\text { percentage }(\%)\end{array}$ \\
\hline Ala (A) & 11 & $6.7 \%$ \\
\hline $\operatorname{Arg}(\mathrm{R})$ & 4 & $2.4 \%$ \\
\hline $\operatorname{Asn}(\mathrm{N})$ & 6 & $3.6 \%$ \\
\hline Asp (D) & 14 & $8.5 \%$ \\
\hline C0ys (C) & 7 & $4.2 \%$ \\
\hline Gln (Q) & 5 & $3.0 \%$ \\
\hline Glu (E) & 6 & $3.6 \%$ \\
\hline His $(\mathrm{H})$ & 5 & $3.0 \%$ \\
\hline Ile (I) & 6 & $3.6 \%$ \\
\hline Leu (L) & 19 & $11.5 \%$ \\
\hline Lys (K) & 26 & $15.8 \%$ \\
\hline $\operatorname{Met}(\mathrm{M})$ & 6 & $3.6 \%$ \\
\hline Phe (F) & 6 & $3.6 \%$ \\
\hline Pro $(\mathrm{P})$ & 6 & $3.6 \%$ \\
\hline Ser (S) & 8 & $4.8 \%$ \\
\hline Thr (T) & 6 & $3.6 \%$ \\
\hline $\operatorname{Trp}(\mathrm{W})$ & 0 & $0.0 \%$ \\
\hline Tyr (Y) & 5 & $3.0 \%$ \\
\hline Val (V) & 12 & $7.3 \%$ \\
\hline
\end{tabular}

Table 1. Amino acid composition of D7r1 


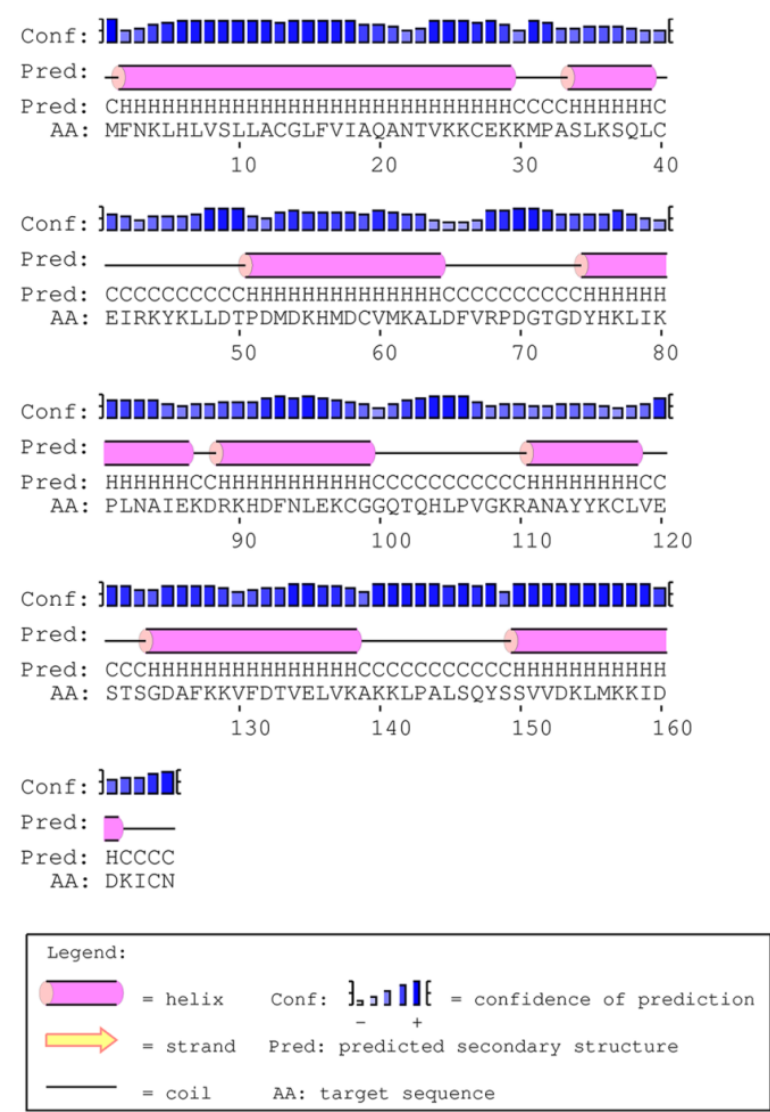

Figure 1. Secondary structure prediction of D7r1 by PSIPRED server

The protein sequence or primary structure decides the function and stability of the protein. Therefore, it was important to analyze the amino acid sequence which provides basic information about $\mathrm{D} 7 \mathrm{r} 1$ protein. The physio-chemical parameters of $\mathrm{D} 7 \mathrm{r} 1$ protein were determined by ProtParam tool of ExPASy server. The amino acid composition of $\mathrm{D} 7 \mathrm{r} 1$ is given in Table 1 . The calculated isoelectric point for $\mathrm{D} 7 \mathrm{r} 1$ was 9.24 which suggest the presence of a high number of charged residues. The estimated molecular weight of $\mathrm{D} 7 \mathrm{r} 1$ is $18,666 \mathrm{Da}$ and an aliphatic index [44] of 86.85. The extinction coefficient [45] of protein (at $280 \mathrm{~nm}$ in measured in water) assuming all pairs of Cys residues from cysteines was $7825 \mathrm{M}^{-1} \mathrm{~cm}^{-1}$ and reduced form was $7450 \mathrm{M}^{-1} \mathrm{~cm}^{-1}$. The instability index [46] of D7r1 was computed to be 24.38 , which clearly implies that the generated 3D model was more stable because an instability index more than 40 is considered unstable [47]. The stability of protein was further confirmed by a Grand average of hydropathicity (GRAVY) value of -0.353 . The secondary structural elements were analyzed by Self-Optimized Prediction Method (SOPMA) [17]. SOPMA analysis shows that D7r1 structure was completely dominated by alpha helix (above 60\%) which further followed by random coil (33\%), and extended strand (7\%). The schematic diagram of secondary structure of D7r1 as generated by PSIPRED server and given in Figure 1.

\subsection{Tertiary Structure Prediction}

The protein sequence of D7r1 (165aa, UniProtKB accession number: Q9UB30) was fetched from Uniprot database [48] in FASTA format. The template based modeling also called homology modeling requires experimentally determined protein structure as a template. The most appropriate template for homology modeling was determined by BLASTp search against the PDB database [49]. The crystal structure of D7r4 (PDB ID: 2PQL) from Anopheles gambiae at $2.2 \AA$ resolution [10] was selected as a template based on the maximum identity score and lower $e$ value. The $\mathrm{D} 7 \mathrm{r} 1$ protein and template $2 \mathrm{PQL}$ showed $55 \%$ sequence identity and $84 \%$ query coverage with an $e$ value of 3e-54, which meets the criteria for choosing a suitable template. Clustal Omega was used to perform an alignment between the target and template protein sequences. Structural modeling was performed by MODELLER (9v11) which generates ten models out of which the best structure is chosen based on the lowest DOPE score (-16487.28906).

The structural features of $\mathrm{D} 7 \mathrm{r} 1$ model were similar to other $\mathrm{D} 7 \mathrm{r}$ protein family members and the predicted model adopted all structural features from the $\mathrm{D} 7 \mathrm{r} 4$ template. The superimposition of model D7r1 and template 2PQL shows that the generated model quality was good with a least RMSD value of $0.117 \AA$. The sequence and structural alignment between the target and template protein reveals that similar active site residues involved in the binding pocket formation (Figure 2). The predicted tertiary structure of D7r1 contains eight helices $\alpha 1$ to $\alpha 8$ which are connected by several loops and the modeled structure given in Figure 3. Among eight helices, $\alpha 5$ and $\alpha 7$ helices found to be larger helices with the helical lengths of $23.97 \AA$ and $26.99 \AA$ respectively; whereas, $\alpha 1$ and $\alpha 4$ helices predicted as small helices with helical lengths of $9.69 \AA$ and $15.92 \AA$ respectively. The N-terminal region of $\mathrm{D} 7 \mathrm{r} 1$ has adopted long loop region (M1-T23) with a length of $18.29 \AA$. The number of $\alpha$ helices and their corresponding amino acid residues with their helical length is given in Table 2. 
D7r1

D $7 r 4$

D $7 r 1$

D7r 4

D7r1

D7r 4

Figure 2. Sequence alignment between $\mathrm{D} 7 \mathrm{r} 1$ and template (2PQL). Active site forming residues are indicated in red color and showed by black arrows.

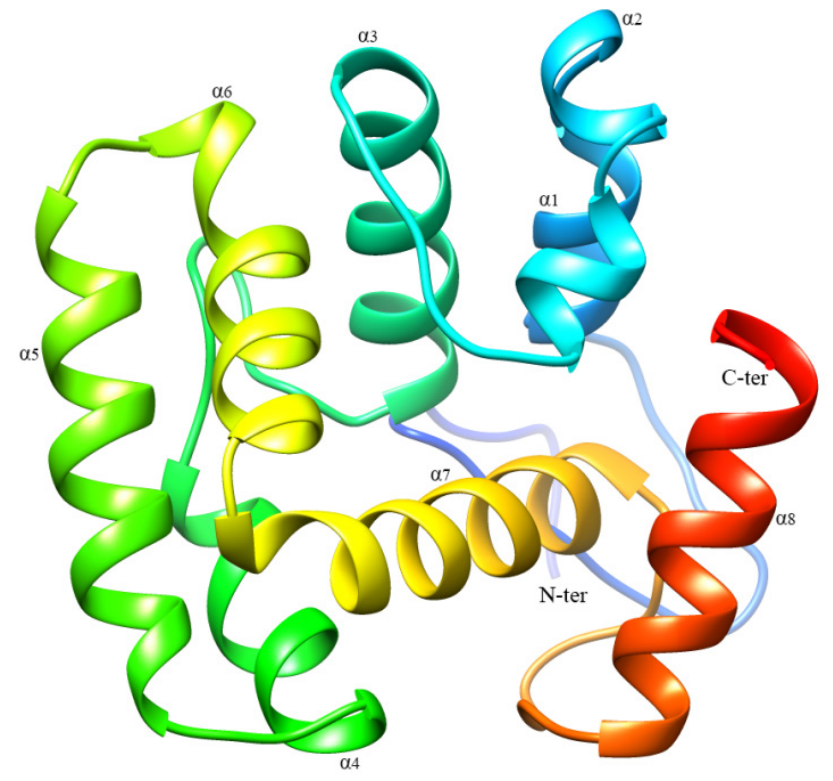

Figure 3. The predicted three dimensional structure of D7r1

Table 2. Number of alpha helices with their helical length $(\AA)$

\begin{tabular}{|c|c|c|c|c|c|}
\hline Helix & Start a.a & End a.a & No.of residues & Helical length $(\AA)$ & Sequence \\
\hline 1 & Val24 & Lys30 & 6 & 9.69 & VKKCEK \\
\hline 2 & Ala33 & Lys44 & 12 & 18.14 & ASLKSQLCEIRK \\
\hline 3 & Pro51 & Leu64 & 14 & 19.69 & PDMDKHMDCVMKAL \\
\hline 4 & Tyr75 & Ile85 & 11 & 15.92 & YHKLIKPLNAI \\
\hline 5 & Arg89 & His104 & 16 & 23.97 & RKHDFNLEKCGGQTQH \\
\hline 6 & Val107 & Glu120 & 14 & 20.38 & VGKRANAYYKCLVE \\
\hline 7 & Thr122 & Ala139 & 18 & 26.99 & TSGDAFKKVFDTVELVKA \\
\hline 8 & Val151 & Ile163 & 13 & 20.56 & VVDKLMKKIDDKI \\
\hline
\end{tabular}




\subsection{Structure Validation of D7r1}

The structural validation and reliability of predicted model of D7r1 was carried out by several online tools such as PROCHECK, ERRAT, Verify-3D and RAMPAGE. A Ramachandran plot was built by RAMPAGE describes that 96.9\% (158 aa) of residues fall in favoured region, 3.1\% (5 aa) of residues fall into the allowed region and none of the residues fall in the disallowed region which reveals the precision of dihedral angles and backbone in three dimensional space (Figure 4). In addition, the quality of modeled structure was evaluated based on the energetic and empirical methods by Verify-3D, which produces average data points for each residue. In this case, a Verify-3D score of D7r1 protein model was $70.91 \%$. ERRAT algorithm validates the reliability of the modeled structure with an ERRAT score of 92.10. In addition, PROSA web server was used to check the quality of the model in terms of $\mathrm{Z}$ score, a measure of overall model quality and confirms whether the target protein was within the acceptable range of X-ray and NMR studies. The $Z$ score for the D7r1 protein model was found to be -5.61 which suggest a better quality model (Figure 5). The Q mean server was used to examine the model quality by comparing all available reference structures which are experimentally solved. This server result in six structural parameters including C_beta interaction energy -43.85 (Z-score: -1.77), all-atom pairwise energy -3482.66 (Z-score:-1.29), solvation energy -2.52 (Z-score:-1.88), torsion angle energy -24.36 (Z-score: -1.65$)$, secondary structure agreement $75.8 \%$ (Z-score: -1.03 ), and solvent accessibility agreement $77.6 \%$ (Z-score: -0.15 ), which further signifies a model of better quality. The quality of computationally modeled D7r1 structure was further validated by PROSESS web server. The PROSESS score for covalent bond quality (6.5), non-covalent/packing quality (6.5) and torsional quality (6.5) prove that the predicted D7r1 model in high quality. Figure 6A represents the overall quality factor and Figure $6 \mathrm{~B}$ shows the number of parameters with outlier for each residue.

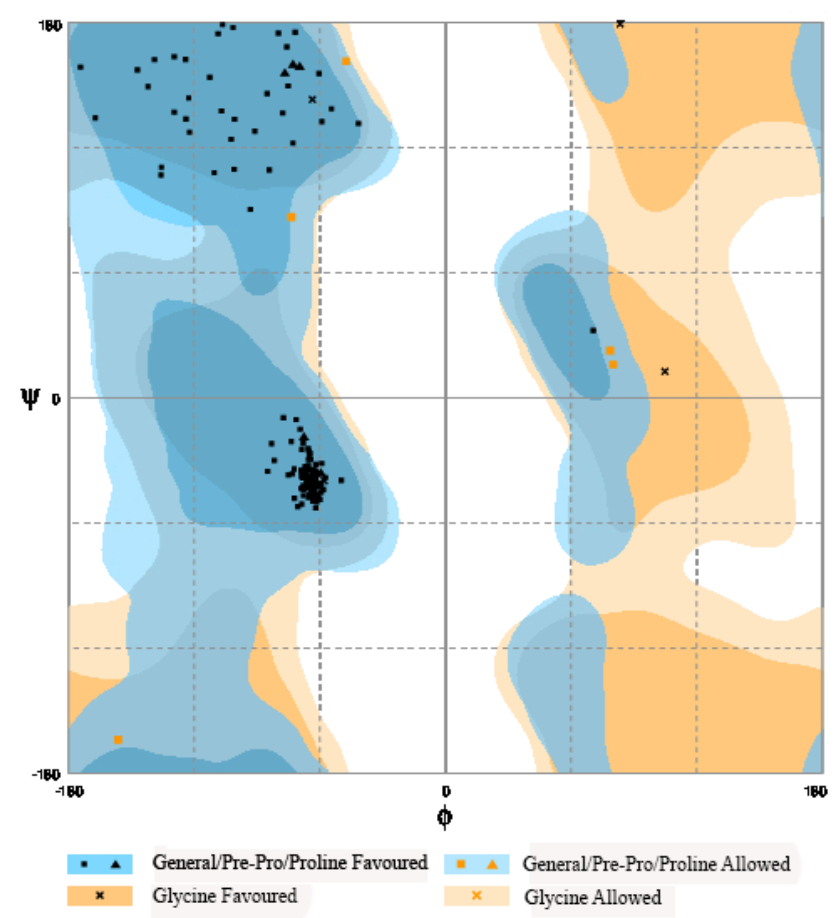

Figure 4. Ramachandran plot of the modeled D7r1 built by RAMPAGE

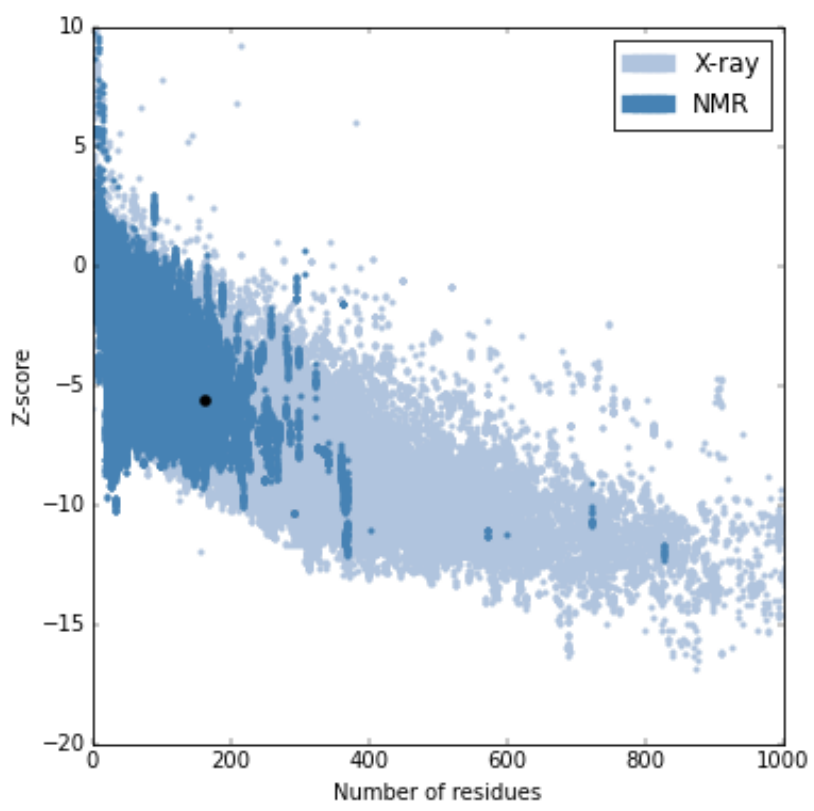

Figure 5. Validation of D7r1 model by Z-score of ProSA 
(A)

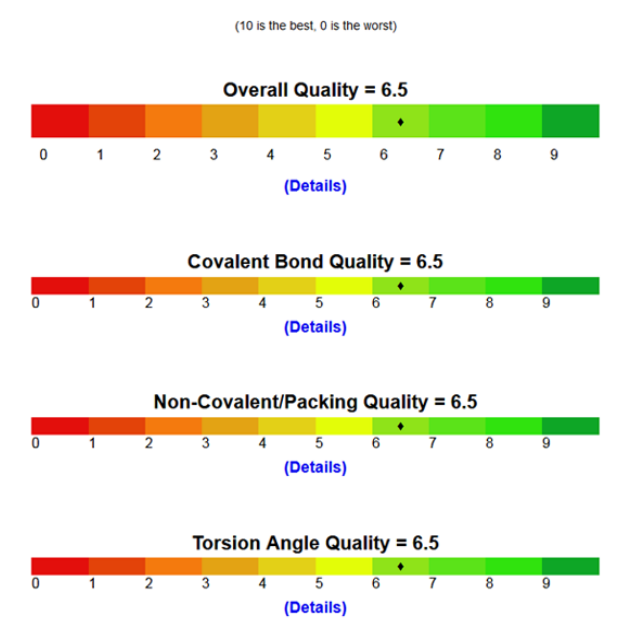

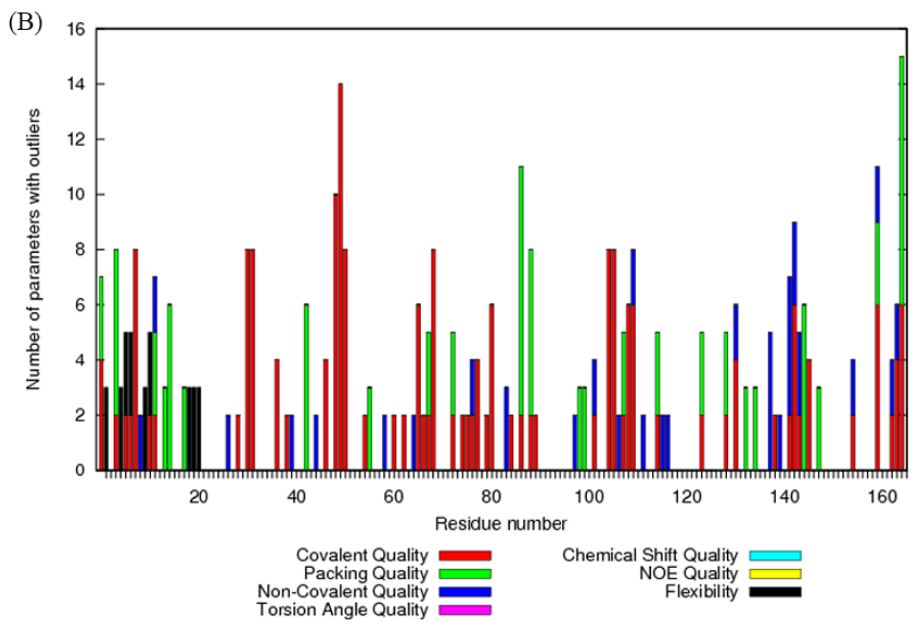

Figure 6. Quantitative evaluation of D7r1 by PROSESS server. (A) Overall quality factor (B) Number of parameters with outlier for each residue.

\subsection{Helical Nature of D7r1}

The phi $(\Phi)$ and psi $(\Psi)$ values obtained from PROSESS web server is used as a measure to evaluate the helical nature of D7r1. For each helix ( $\alpha 1$ to $\alpha 8)$, the phi $(\Phi)$ and psi $(\Psi)$ values are retrieved from the residual report of PROSESS web server. The backbone dihedral angles $\Phi$ and $\Psi$ fall in a narrow region of the Ramachandran plot, with $\Phi$ between $-95^{\circ}$ and $-35^{\circ}$, and $\Psi$ between $-15^{\circ}$ and $-70^{\circ}$ [50]. The sum of $\Phi$ and $\Psi$ angles on adjacent residues decides the helical form of protein, in which the angle for $\alpha$-helices is approximately $-105^{\circ}$. The sum of above mentioned angle for $\pi$-helix and $3_{10}$-helix is $-75^{\circ}$ and $-130^{\circ}$ respectively [51]. The average sum values of $\Phi$ and $\Psi$ angles for each alpha helices such as $\alpha 1$ $\left(-99.97^{\circ}\right), \alpha 2\left(-98.25^{\circ}\right), \alpha 3\left(-103.52^{\circ}\right), \alpha 4\left(-108.67^{\circ}\right), \alpha 5$ $\left(-103.74^{\circ}\right), \alpha 6\left(-104.79^{\circ}\right), \alpha 7\left(-102.33^{\circ}\right)$ and $\alpha 8\left(-106.07^{\circ}\right)$ were calculated which clearly proves the alpha helical nature of $\mathrm{D} 7 \mathrm{r} 1$ protein.

\subsection{Simulation of $D 7 \mathbf{r} 1$}

In general, MD simulation has been used to refine and understand the stability of structure predicted by homology modeling approach [52]. Herein, the assessment of the stability of the modeled D7r1 was carried out by equilibrating the protein for a time scale of $50 \mathrm{~ns}$. The root-mean-square deviation(RMSD) as a standard to evaluate the structural stability of the protein for the protein backbone atoms. The backbone RMSD profile shows the D7r1 attains equilibrium after the 15,000 ps and remains stable for rest of the simulation period (Fig.7A). Meanwhile, the root mean square fluctuation (RMSF) of D7r1 was generated to distinguish the mobility of each residue and depicted in Fig.7B. The RMSF fluctuation of D7r1 shows that it has two flexible regions (F1 and F2). The residues present in N-terminal loop region (A12-V17) shows gradual fluctuation with a value of $0.39 \mathrm{~nm}$ and are recognized as an flexible region (F1) of D7r1. Whereas, the high flexible region (F2) was observed in residues between K140-Q147 with an RMS fluctuation of $0.47 \mathrm{~nm}$. The compactness of the D7r1 was ensured by calculating the Radius of gyration $\left(R_{g}\right)$ and shown in Fig.7C. The potential energy graph in Fig.7D shows that trajectory with minimum potential energy conformation was observed at 15140 ps. Further, the minimum and maximum potential energy of $\mathrm{D} 7 \mathrm{r} 1$ was calculated as -768433.31 and -761988.31 respectively, which explains that the predicted model were stable in terms of energetic behavior during the MD simulation. Accordingly, the minimized representative structure was retrieved from equilibrated part of trajectory by g_dump command in GROMACS suite. The minimized D7r1 model was subjected to structural validation by the same above mentioned tools. 
(A)

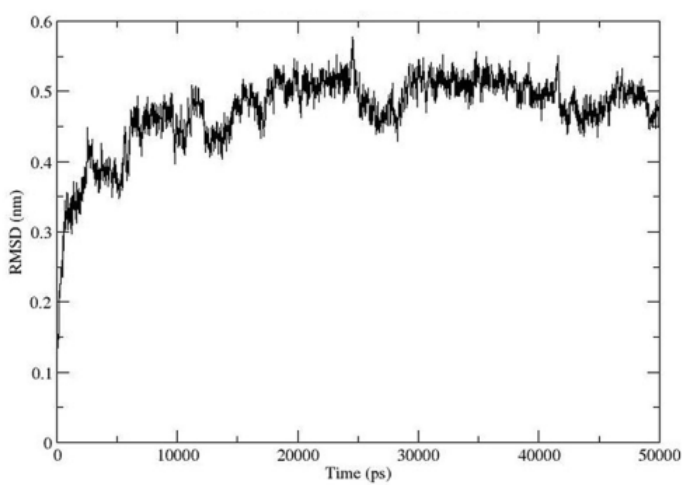

(B)
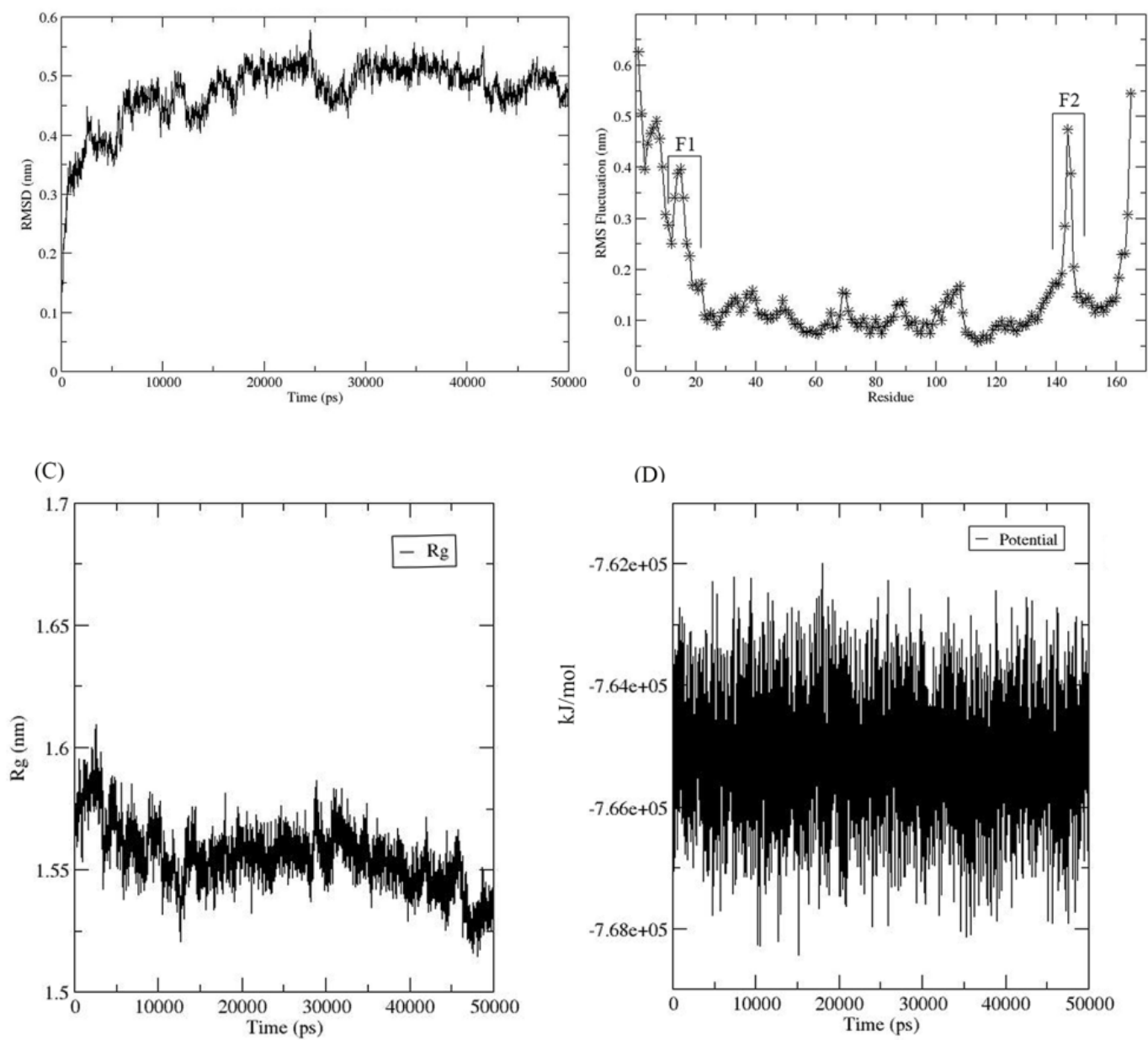

(D)

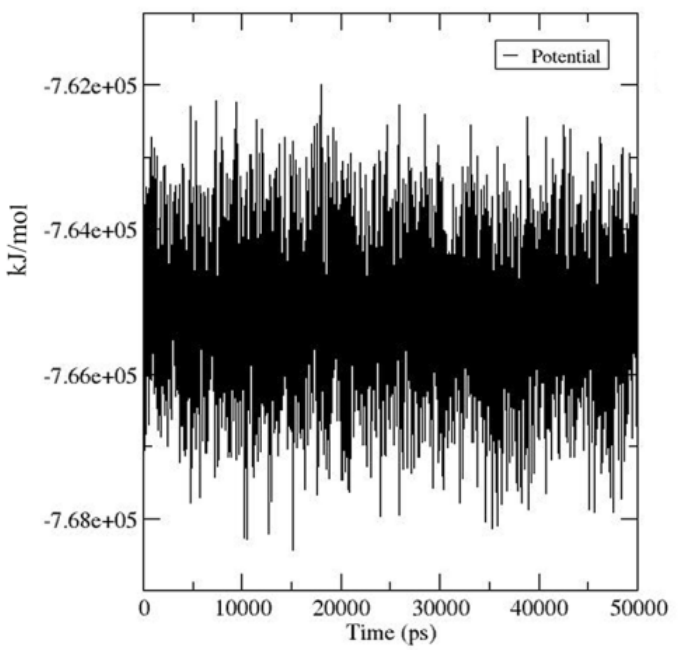

Figure 7. The results of molecular dynamics simulation. (A) Backbone RMSD of D7r1 during 50ns. (B) Backbone RMS fluctuation of D7r1 depicting two flexible regions. (C) Showing Radius of gyration (Rg) of D7r1 (D) Showing Potential energy of D7r1

\subsection{Principal Component and FEL Analysis of D7r1}

In order to understand the structural and conformational stability of $\mathrm{D} 7 \mathrm{r} 1$, the principal component analysis and free energy landscape analysis were performed. The first step involves generation of covariance matrix of protein alpha carbon $(\mathrm{C} \alpha)$, in which 1659 protein elements were used with sum of eigenvalues to be $5.991 \mathrm{~nm}^{2}$. The top eigenvectors responsible for approximately $90 \%$ of the internal motions of the protein and therefore the first five, tenth and the twentieth projections from the trajectory were retrieved and projected onto the eigenvectors against time during the 50ns simulation period (Fig.8). Further FEL plot was generated based on the cosine content values $(<1)$ of principal components (PCs), as they are found to be capturing the functional behavior of the protein and an indicator of good simulation sampling. In our study, the FEL plot was generated for $\mathrm{D} 7 \mathrm{r} 1$ trajectory using principal components (PC1 and $\mathrm{PC} 2$ ) to observe the conformational changes during the course of the simulation. The FEL plot shows three different energy clusters, which corresponds to different conformations of $\mathrm{D} 7 \mathrm{r} 1$ protein. The energy cluster
$\mathrm{C} 1, \mathrm{C} 2$ and $\mathrm{C} 3$ represent the minimized structures of $\mathrm{D} 7 \mathrm{r} 1$ at $18 \mathrm{~ns}, 32 \mathrm{~ns}$ and $40 \mathrm{~ns}$ respectively (Fig.9A). The FEL contour map in Fig.9B also used to visualize the representative structure with minimal energy. In addition, the minimized structures from FEL analysis were retrieved and superimposed with initial predicted structure, which shows the RMSD deviation of $3.462 \AA, 3.380 \AA$ and $2.956 \AA$ for $18 \mathrm{~ns}, 32 \mathrm{~ns}$ and $40 \mathrm{~ns}$ representative structures respectively. Further, low energy clusters were subjected to structural validation by the RAMPAGE and the Ramachandran plot given in Fig.10. The Ramachandran plot built by RAMPAGE shows that residues falling in most favored regions found to be $86.4 \%, 84 \%$ and $84 \%$, for $18 \mathrm{~ns}, 32 \mathrm{~ns}$ and 40 ns respectively. Similarly, residues falling in disallowed regions found to be $3.1 \%, 3.1 \%$ and $2.5 \%$ for $18 \mathrm{~ns}, 32 \mathrm{~ns}$ and 40ns respectively, meanwhile the ERRAT values calculated as $94.94,93.63$ and 88.81 respectively. These validation results provide insights into the reliability of modeled structure. Further, in view of ERRAT and Ramachandran plot, 18ns refined structure considered as a good quality structure. 


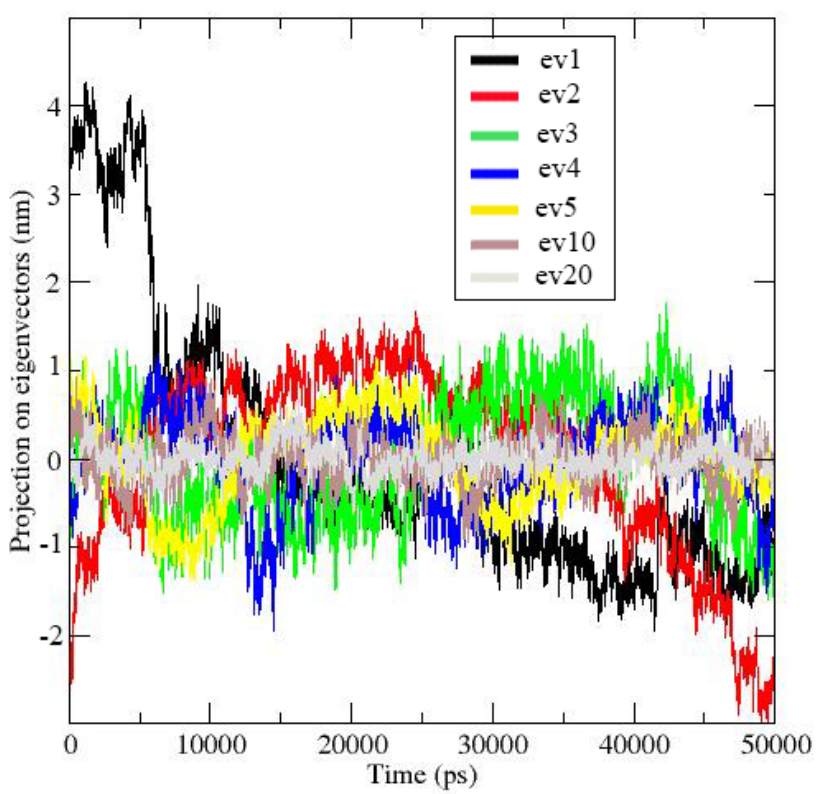

Figure 8. Principal component analysis (PCA) of D7r1. The motions along with the first five, tenth and twentieth eigenvectors with their respective color code during the $50 \mathrm{~ns}$ simulation.

\subsection{Active Site Prediction}

The active site of $\mathrm{D} 7 \mathrm{r} 1$ as predicted from both computational and literature studies. The $\mathrm{COACH}$ server, a meta-server, which is combined of several online servers which includes COFACTOR, ConCavity and S-SITE were used to predict the residues which are involved in the binding pocket formation (Table.3). Further, the sequence homology and structure conservancy between $\mathrm{D} 7 \mathrm{r} 1$ and template $2 \mathrm{PQL}$ shows high degree of consistent [10]. Hence, the active site residues of Anopheles gambiae $\mathrm{D} 7 \mathrm{r} 4$ and ligand tryptamine $\left(\mathrm{C}_{10} \mathrm{H}_{12} \mathrm{~N}_{2}\right)$ were retrieved by submitting $2 \mathrm{PQL}$ to PDB sum [53] server. Clustal Omega [20] tool used to align the sequence of the target and template protein and the corresponding active site residues were predicted. The amino acid residues Ile42, Arg43, Tyr45, His56, Met57, Val60, Phe131, and Met156 are identified as active site residues.

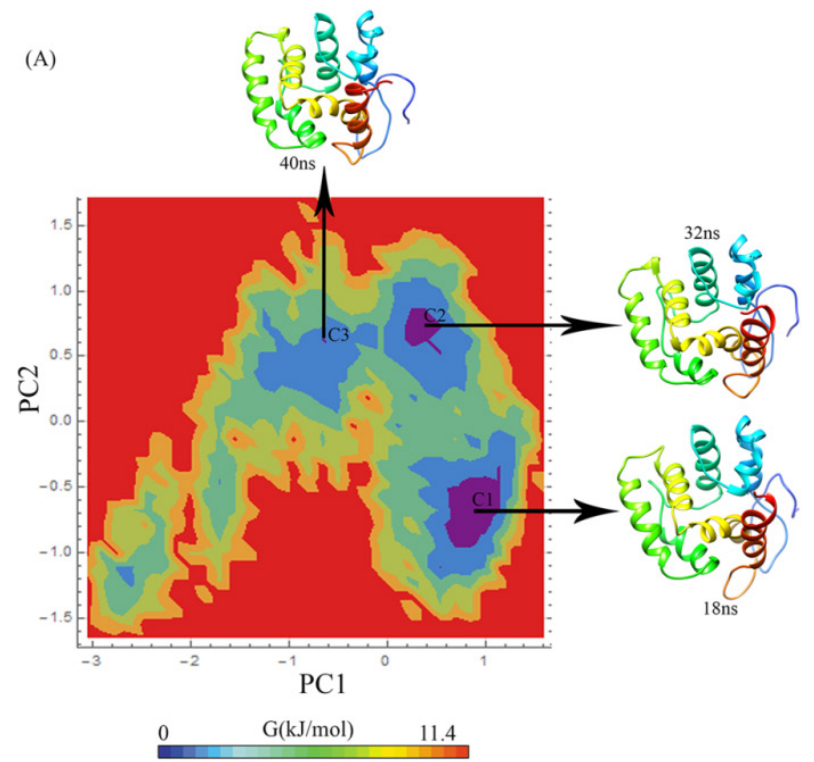

(B)

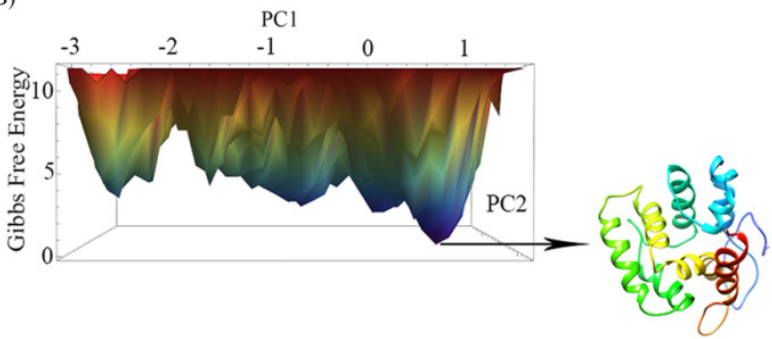

Figure 9. PCA based Free energy landscape (FEL). (A) FEL representative energy cluster are shown as $\mathrm{C} 1, \mathrm{C} 2$ and $\mathrm{C} 3$ for $18 \mathrm{~ns}, 32 \mathrm{~ns}$ and $40 \mathrm{~ns}$ respectively. Corresponding representative structure are retrieved and displayed accordingly. (B) FEL contour map which depicts lowest energy conformation with their respective structure.

Table 3. Predicted active site residues by COACH, COFACTOR, ConCavity and S-SITE server with their respective C-score.

\begin{tabular}{|c|c|c|c|}
\hline Server Name & Ligand name & Residue Numbers & C-score \\
\hline COACH & SRO & $\begin{array}{c}\text { V24, E28, I42, R43, Y45, H56, M57, V60, Y115, F131, } \\
\text { D132, E135, M156 }\end{array}$ & 0.79 \\
\hline COFACTOR & HSM & I42, R43, Y45, Y115, F131, D132, E135 & 0.56 \\
\hline ConCavity & Cavity 1 & $\begin{array}{c}\text { V24, E28, C40, I42, R43, K44, Y45, H56, M57, V60, } \\
\text { Y115, F131, D132, E135, D160 }\end{array}$ & 0.23 \\
\hline S-SITE & SRO & $\begin{array}{c}\text { V24, E28, I42, R43, Y45, H56, M57, V60, Y115, F131, } \\
\text { D132, E135, M156, D160 }\end{array}$ & 0.48 \\
\hline
\end{tabular}




\subsection{Disordered Region Prediction and Evolutionary Conservation Analysis}

In proteomics, the disorder can be defined as the lack of regular secondary structural elements and high degree of flexibility in the protein structure. The high flexibility of the disordered region in a protein might help to adopt different conformational possibilities. Further, the disordered region contains functional sites or linear motifs which influence the functionality of the protein. Therefore, to identify globular and disorder regions in D7r1 protein sequence GlobPlot were used and it is anticipated that two regions 69-78 and 100-105 are intrinsically disordered within the protein sequence. In X-axis, the blue color coded regions are disordered regions and green colored regions are ordered or globular regions of D7r1 (Fig. 11).
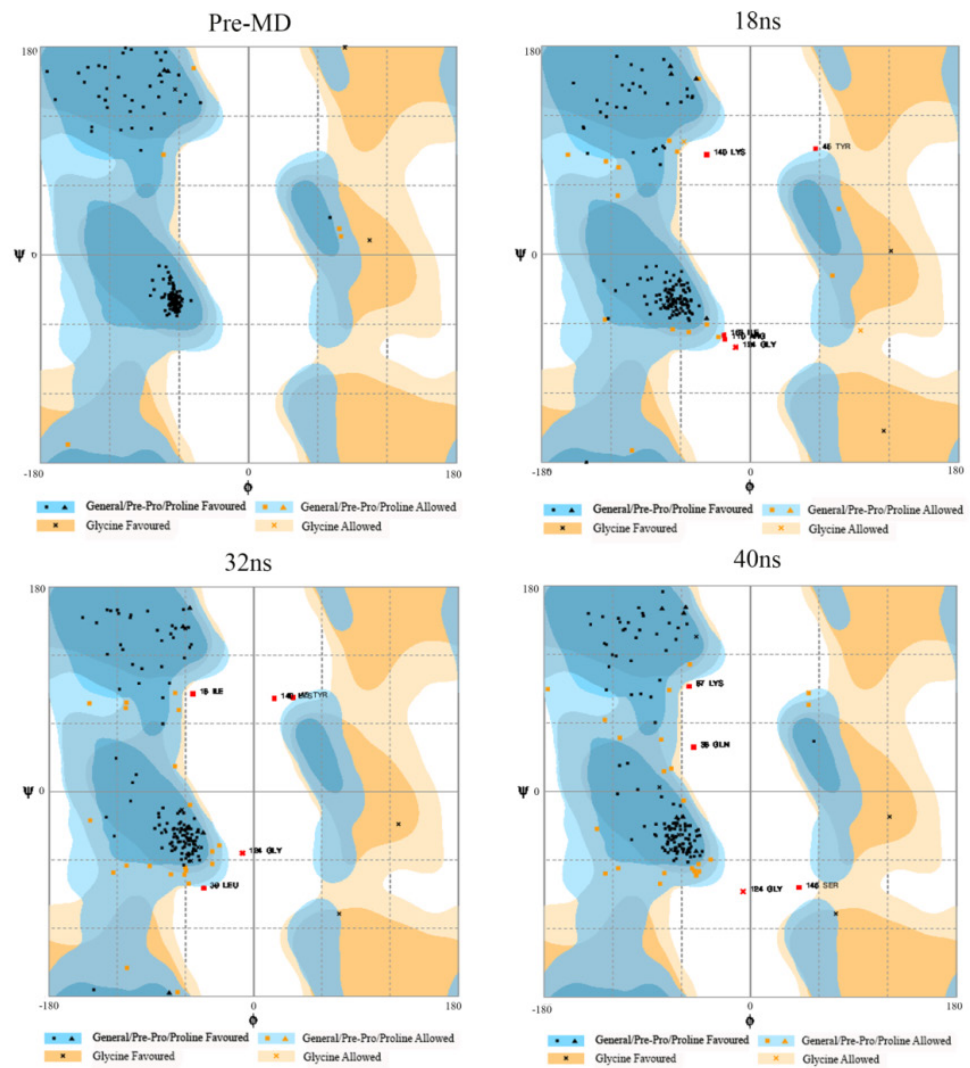

Figure 10. Comparative diagram representing Ramachandran plot of D7r1 of Pre and after MD simulations at 18ns, 32ns and 40ns. The plots were built by RAMPAGE

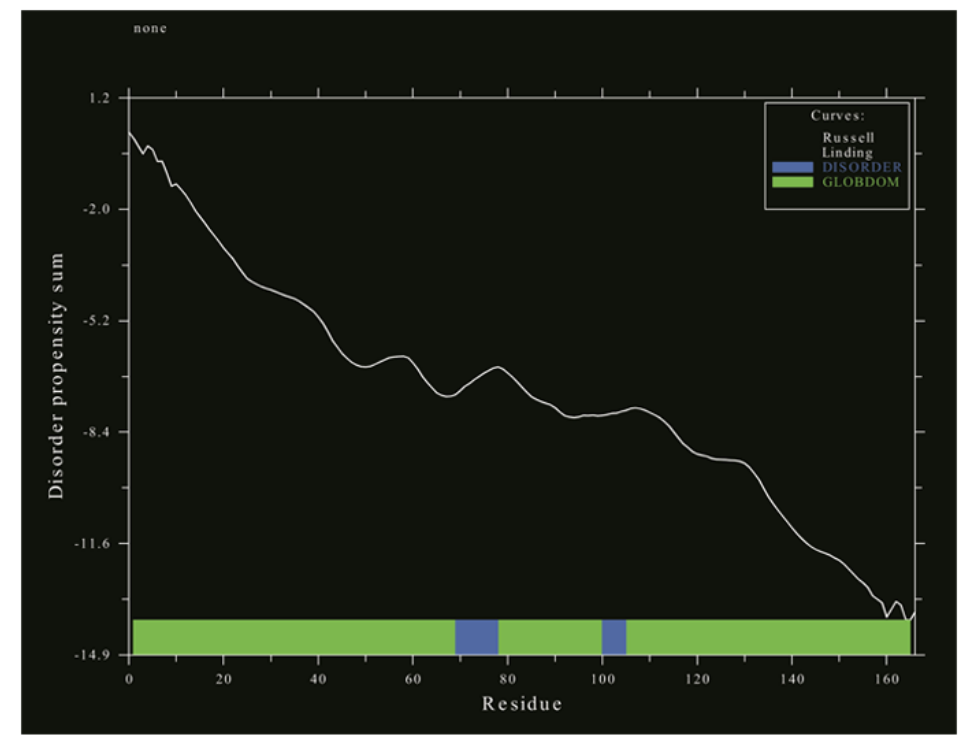

Figure 11. The globular and disordered regions of D7r1 shown in green and blue color respectively 


\section{Conclusions}

Owing to the importance of the saliva protein D7r1 of Anopheles gambiae as an anti-malarial target, the present study reports the in silico structural analysis that providing insights into its structural architecture. In silico analysis has reached to such advancement that results obtained from computational study are much more reliable and comparable to experimental results. The three dimensional structure of D7r1 has been predicted through homology modeling which shows structural similarity to its experimentally determined template structure (PDB ID: 2PQL). Similarly, the predicted D7r1 also contains eight helices connected by several loops which resemble the structural architecture of D7r4. At present, the molecular dynamics simulation study proven to be an essential step in computational biology which provides insight into protein structural stability through RMSD and RMSF plot. In our study, to explore the conformational flexibility and structural transition occur in D7r1, essential dynamics were employed which shows that three cluster basin at different time interval. Accordingly, FEL representative structures at different conformational state were retrieved and validated through Ramachandran plot. Further, similar to D7r4, same active site residues involved in binding pocket formation and opened a gateway to investigate the interacting mechanism of $\mathrm{D} 7 \mathrm{r} 1$ with its suitable ligands and protein interacting partners through molecular docking study. Since, the structural knowledge of macromolecule is an important pre-requisite to understand its functional role. Hence, the predicted D7r1 structure and its active site might be useful in further attempts to elucidate the experimental structures as well as to design new lead molecules acting on novel drug targets considered as a suitable starting point for structure based drug design in the treatment of malaria.

\section{Conflicts of Interests}

Authors declare no conflicts of interest.

\section{Author Contribution}

Manikandan Jayaraman and Chayanika Goswami contributed equally.

\section{Acknowledgements}

Krishna Ramadas thanks Indian Council of Medical Research (ICMR), Government of India for providing financial assistance (No.BIC/12(27)2012) to carry out the research work. Manikandan Jayaraman

(F./2016-17/NFO-2015-17-OBC-PON-35843/Feb2016) and Guneswar Sethi

(F./2014-15/RGNF-2014-15-SC-ORI-74382) thanks UGC for Rajiv Gandhi National Fellowship to pursue their PhD degree.

\section{REFERENCES}

[1] Gallup J.L., Sachs J.D. The economic burden of malaria. Am J Trop Med Hyg. 2001, 64, 85-96.

[2] "World Health Organization." [Online]. Available: http://www.who.int/mediacentre/factsheets/fs094/en/.

[3] Calvo E., DeBianchi A.G., James A.A., Marinotti O. The major acid soluble proteins of adult female Anopheles darlingi salivary glands include a member of the D7-related family of proteins. Insect Biochem Mol Biol. 2002, 32, 1419-27.

[4] Tsitsanou K.E., Thireou T., Drakou C.E., Koussis K., Keramioti M.V., Leonidas D.D., Eliopoulos E., Iatrou K., Zographos S.E. Anopheles gambiae odorant binding protein crystal complex with the synthetic repellent DEET: Implications for structure-based design of novel mosquito repellents. Cell Mol Life Sci. 2012, 69(2), 283-97.

[5] Manguin S. Biodiversity of Malaria in the World. Montrouge, France: John Libbey Eurotext; 2008.

[6] Hallem E.A., Nicole Fox A., Zwiebel L.J., Carlson J.R. Olfaction: mosquito receptor for human-sweat odorant. Nature. 2004, 427, 212-213.

[7] Ribeiro J.M., Valenzuela J.G. Purification and cloning of the salivary peroxidase/catechol oxidase of the mosquito Anopheles albimanus. J Exp Biol. 1999, 202, 809-16.

[8] Choumet V., Carmi-Leroy A., Laurent C., Lenormand P., Rousselle J.C., Namane A., Roth C., Brey P.T. The salivary glands and saliva of Anopheles gambiae as an essential step in the Plasmodium life cycle: A global proteomic study. Proteomics. 2007, 7, 3384-94.

[9] Kalume D.E., Okulate M., Zhong J., Reddy R., Suresh S., Deshpande N., Kumar N., Pandey A. A proteomic analysis of salivary glands of female Anopheles gambiae mosquito. Proteomics. 2005, 5, 3765-77.

[10] Mans B.J., Calvo E., Ribeiro J.M.C., Andersen J.F. The crystal structure of $\mathrm{D} 7 \mathrm{r} 4$, a salivary biogenic amine-binding protein from the malaria mosquito Anopheles gambiae. J Biol Chem. 2007, 282, 36626-33.

[11] Sor-suwan S., Jariyapan N., Roytrakul S., Paemanee A., Phumee A., Phattanawiboon B., Intakhan N., Chanmol W., Bates P.A., Saeung A., Choochote W. Identification of salivary gland proteins depleted after blood feeding in the malaria vector Anopheles campestris-like mosquitoes (Diptera: Culicidae). PLoS One. 2014, 9, e90809.

[12] Rawal R, Vijay S, Kadian K, Singh J, Pande V, Sharma A. Towards a Proteomic Catalogue and Differential Annotation of Salivary Gland Proteins in Blood Fed Malaria Vector Anopheles culicifacies by Mass Spectrometry. PLoS One. 2016, 11, e0161870.

[13] Isawa H., Orito Y., Iwanaga S., Jingushi N., Morita A., Chinzei Y., Yuda M. Identification and characterization of a new kallikrein-kinin system inhibitor from the salivary glands 
of the malaria vector mosquito Anopheles stephensi. Insect Biochem Mol Biol. 2007, 37, 466-77.

[14] Calvo E., Mans B.J., Andersen J.F., Ribeiro J.M.C. Function and Evolution of a Mosquito Salivary Protein Family. J Biol Chem. 2006, 281, 1935-1942.

[15] Colovos C., Yeates T.O. Verification of protein structures: Patterns of nonbonded atomic interactions. Protein Sci. 1993, $2,1511-9$.

[16] Buchan D.W.A., Minneci F., Nugent T.C.O., Bryson K., Jones D.T. Scalable web services for the PSIPRED Protein Analysis Workbench. Nucleic Acids Res. 2013, 41.

[17] Guermeur Y., Geourjon C., Gallinari P., Deléage G. Improved performance in protein secondary structure prediction by inhomogeneous score combination. Bioinformatics. 1999, 15, 413-21.

[18] “GlobPlot." [Online]. Available: http://globplot.embl.de/.

[19] Eswar N., John B., Mirkovic N., Fiser A., Ilyin V.A., Pieper U., Stuart A.C., Marti-Renom M.A., Madhusudhan M.S., Yerkovich B., Sali A. Tools for comparative protein structure modeling and analysis. Nucleic Acids Res. 2003, 31, 3375-80.

[20] Sievers F., Wilm A., Dineen D., Gibson T.J., Karplus K., Li W., Lopez R., McWilliam H., Remmert M., Söding J., Thompson J.D., Higgins D.G. Fast, scalable generation of high-quality protein multiple sequence alignments using Clustal Omega. Mol Syst Biol. 2011, 7, 539.

[21] "Pymol." [Online]. Available: http://www.pymol.org/.

[22] Pettersen E.F., Goddard T.D., Huang C.C., Couch G.S., Greenblatt D.M., Meng E.C., Ferrin T.E. UCSF Chimera-A Visualization System for Exploratory Research and Analysis. J Comput Chem. 2004, 25, 1605-12.

[23] Lovell SC, Davis IW, Iii WBA, Bakker PIW De, Word JM, Prisant MG, Richardson JS, Richardson DC. Structure Validation by $\mathrm{C} \alpha$ Geometry : $\varphi, \psi$ and $\mathrm{C} \beta$ Deviation. 2003, 450, 437-50.

[24] "SAVESERVER." [Online]. Available: https://services.mbi.ucla.edu/SAVES/.

[25] Laskowski R.a., MacArthur M.W., Moss D.S., Thornton J.M. PROCHECK: a program to check the stereochemical quality of protein structures. J Appl Crystallogr. 1993, 26, 283-91.

[26] Lüthy R., Bowie J.U., Eisenberg D. Assessment of protein models with three-dimensional profiles. Nature. 1992, 356, $83-5$.

[27] Benkert P., Künzli M., Schwede T. QMEAN server for protein model quality estimation. Nucleic Acids Res. 2009, 37, W510-4.

[28] Wiederstein M., Sippl M.J. ProSA-web: Interactive web service for the recognition of errors in three-dimensional structures of proteins. Nucleic Acids Res. 2007, 35.

[29] Berjanskii M., Liang Y., Zhou J., Tang P., Stothard P., Zhou Y., Cruz J., MacDonell C., Lin G., Lu P., Wishart D.S. PROSESS: A protein structure evaluation suite and server. Nucleic Acids Res. 2010, 38.

[30] Willard L. VADAR: a web server for quantitative evaluation of protein structure quality. Nucleic Acids Res. 2003, 31,
$3316-9$.

[31] Van Der Spoel D., Lindahl E., Hess B., Groenhof G., Mark A.E., Berendsen H.J.C. GROMACS: Fast, flexible, and free. Vol. 26, Journal of Computational Chemistry. 2005, 1701-18.

[32] Hess B., Kutzner C., Van Der Spoel D., Lindahl E. GRGMACS 4: Algorithms for highly efficient, load-balanced, and scalable molecular simulation. J Chem Theory Comput. 2008, 4, 435-47.

[33] Chiu S.W., Pandit S.A., Scott H.L., Jakobsson E. An improved united atom force field for simulation of mixed lipid bilayers. J Phys Chem B. 2009, 113, 2748-63.

[34] Kawata M., Nagashima U. Particle mesh Ewald method for three-dimensional systems with two-dimensional periodicity. Chem Phys Lett. 2001, 340, 165-72.

[35] Miyamoto S., Kollman P.A. Settle: An analytical version of the SHAKE and RATTLE algorithm for rigid water models. J Comput Chem. 1992, 13, 952-62.

[36] Martonák R., Laio A., Parrinello M. Predicting crystal structures: the Parrinello-Rahman method revisited. Phys Rev Lett. 2003, 90, 75503.

[37] Amadei A., Linssen A.B., de Groot B.L., van Aalten D.M., Berendsen H.J. An efficient method for sampling the essential subspace of proteins. J Biomol Struct Dyn. 1996, 13, 615-25.

[38] Wan H., Chang S., Hu J.P., Tian Y.X., Tian X.H. Molecular dynamics simulations of ternary complexes: Comparisons of LEAFY protein binding to different DNA motifs. J Chem Inf Model. 2015, 55, 784-94.

[39] Ivanov P.M. Conformations of some large-ring cyclodextrins derived from conformational search with molecular dynamics simulations and principal component analysis. J Phys Chem B. 2010, 114, 2650-9.

[40] Maisuradze G.G., Liwo A., Scheraga H.A. Relation between free energy landscapes of proteins and dynamics. J Chem Theory Comput. 2010, 6, 583-95.

[41] Roy A., Yang J., Zhang Y. COFACTOR: An accurate comparative algorithm for structure-based protein function annotation. Nucleic Acids Res. 2012, 40(W1).

[42] Capra J.A., Laskowski R.A., Thornton J.M., Singh M., Funkhouser T.A. Predicting protein ligand binding sites by combining evolutionary sequence conservation and 3D structure. PLoS Comput Biol. 2009, 5, e1000585

[43] Yang J., Roy A., Zhang Y. Protein-ligand binding site recognition using complementary binding-specific substructure comparison and sequence profile alignment. Bioinformatics. 2013, 29, 2588-95.

[44] Ikai A. Thermostability and aliphatic index of globular proteins. J Biochem. 1980, 1898, 1895-8.

[45] Gill S.C., von Hippel P.H. Calculation of protein extinction coefficients from amino acid sequence data. Analytical biochemistry. 1989, 182, 319-26.

[46] Guruprasad K., Reddy B.V.B., Pandit M.W. Correlation between stability of a protein and its dipeptide composition: A novel approach for predicting in vivo stability of a protein from its primary sequence. Protein Eng Des Sel. 1990, 4, 15561. 
[47] Wedemeyer W.J., Welker E., Narayan M., Scheraga H.A. Disulfide bonds and protein folding. Biochemistry. 2000, 39, 4207-16.

[48] "UniProt.” [Online]. Available: http://www.uniprot.org.

[49] “RCSB PDB.” [Online]. Available: http://www.rscb.org/pdb/.

[50] Lesk A. Introduction to protein architecture. Oxford: Oxford University Press; 2001.

[51] Guo Z., Mohanty U., Noehre J., Sawyer T.K., Sherman W.,
Krilov G. Probing the alpa-helical structural stability of stapled p53 peptides: Molecular dynamics simulations and analysis. Chem Biol Drug Des. 2010, 75, 348-59.

[52] Raval A., Piana S., Eastwood M.P., Dror R.O., Shaw D.E. Refinement of protein structure homology models via long, all-atom molecular dynamics simulations. Proteins Struct Funct Bioinforma. 2012, 80, 2071-9.

[53] "PDBsum." [Online]. Available: http://www.ebi.ac.uk/pdbsum. 